\title{
Strategies towards controlling the topology of
}

\section{nonlinear poly(thiophenes)}

\author{
Joost Steverlynck ${ }^{1}$, Frederic Monnaie ${ }^{1}$, Emeline Warniez², \\ Roberto Lazzaroni ${ }^{2}$, Philippe Leclère ${ }^{2 *}$, Guy Koeckelberghs ${ }^{1 *}$ \\ ${ }^{1}$ Laboratory for Polymer Synthesis, KU Leuven, Celestijnenlaan 200F, \\ B-3001 Heverlee (Belgium) \\ ${ }^{2}$ Service de Chimie des Matériaux Nouveaux, Université de Mons (UMONS), \\ B-7000 Mons (Belgium)
}

Keywords: conjugated polymers; supramolecular structures; self-assembly; branched polymers

\begin{abstract}
In this contribution, we report the synthesis of all-conjugated branched poly(thiophenes) with a control over the degree of nonlinearity via two different routes. In the first approach a branched monomer is polymerized using three different catalysts which differentiate in the degree of association to the $\pi$-system of the polymer backbone. In the second approach a copolymerization is performed with the branched and a linear monomer. In a next step the influence of the different degrees of nonlinearity on the optoelectronic properties are investigated by UV-vis and fluorescence spectroscopy. Finally, the thermal behavior, the morphology and the electrical properties at the nanoscale of the material in solid state is studied using DSC and AFM techniques, respectively.
\end{abstract}

\section{INTRODUCTION}

While linear conjugated polymers, like homopolymers and different types of copolymers, have been extensively investigated, the domain of conjugated polymers with nonlinear topologies is still largely unexplored. This nonlinearity can be achieved in 2 different ways. In the case of 
branching the monomer units are always elongated further at 2 different positions. Another possibility is that the elongation occurs in more than one coupling pattern (eg. 2,4 and/or 2,5). (Hyper)branched conjugated polymers are but one example of polymers with an architecture different from linear 1D chains. For graft, mikto-arm and star conjugated polymers, reports are even more scarce. ${ }^{1-8}$ Apart from branched conjugated polymers, there are also reports of branched conjugated oligomers and dendrimers. ${ }^{9-13}$ However, for the former the polymeric nature is not fully exploited, while synthesis of the latter is very time consuming and therefore restricted to small amounts. The (hyper)branched conjugated polymers are not just an academic curiosity, but offer a whole new range of properties and therefore applications compared to their linear counterparts. ${ }^{14-21} \mathrm{In}$ addition, they suffer less from insolubility and $1 \mathrm{D}$ anisotropy related problems. $^{22,23}$ Also for light-emitting devices the performance can be improved using the branched counterparts as a result of their in general worse stacking. Although nonlinear conjugated polymers offer a whole new range of opportunities their synthesis is challenging and most of the synthetic methods for the reported (hyper)branched polymers do not offer a control over the degree of nonlinearity. ${ }^{24}$ This, however, poses a limitation on the optimization of the properties of the final material.

In this work we focus on poly(thiophenes). This material is especially interesting because linear poly(thiophenes) can be polymerized in a controlled way and their high stability and good processability renders them useful for applications..$^{25,26}$ There are several reports ${ }^{27-32}$ on the preparation of hyperbranched poly(thiophenes), but there was only one attempt, by the group of Luscombe ${ }^{32}$, towards the synthesis of branched poly(thiophenes) with control over the branching degree. This was achieved by polymerizing 2-bromo-3-hexylthiophene via direct arylation, adjusting the base and ligand. However, this approach only allows control on a noncontinuous scale and the polymerization proceeds via a step growth mechanism. In this article we report two new approaches towards branched poly(thiophenes) offering control over the 
degree of nonlinearity, while keeping the degree of polymerization constant. Using different techniques the optical, thermal and morphological properties of the materials are studied.

\section{RESULTS AND DISCUSSION}

\section{Synthesis of the monomer and the polymers}

In order to introduce the nonlinearity, a $\mathrm{AB}_{2}$ type bithiophene (5) is chosen. ${ }^{28-32}$ The synthesis of the monomer $\mathbf{5}$ starts by converting 5-bromo-3-hexylthiophene to a Grignard reagent (1) and subsequent Kumada coupling to 3-bromothiophene. The bithiophene (2) is then dibrominated on the 2- and 2'- position with $N$-bromosuccinimide (NBS). Finally, the precursor monomer (4) is obtained by mono iodination on the 5-position using lithium diisopropylamide (LDA) and quenching with $\mathrm{I}_{2}$. By a Grignard metathesis reaction the precursor monomer $\mathbf{4}$ is converted to monomer 5 in situ. Adding monomer 5 to $\mathrm{ZnBr}_{2}$ gives monomer 5’. (Scheme 1)
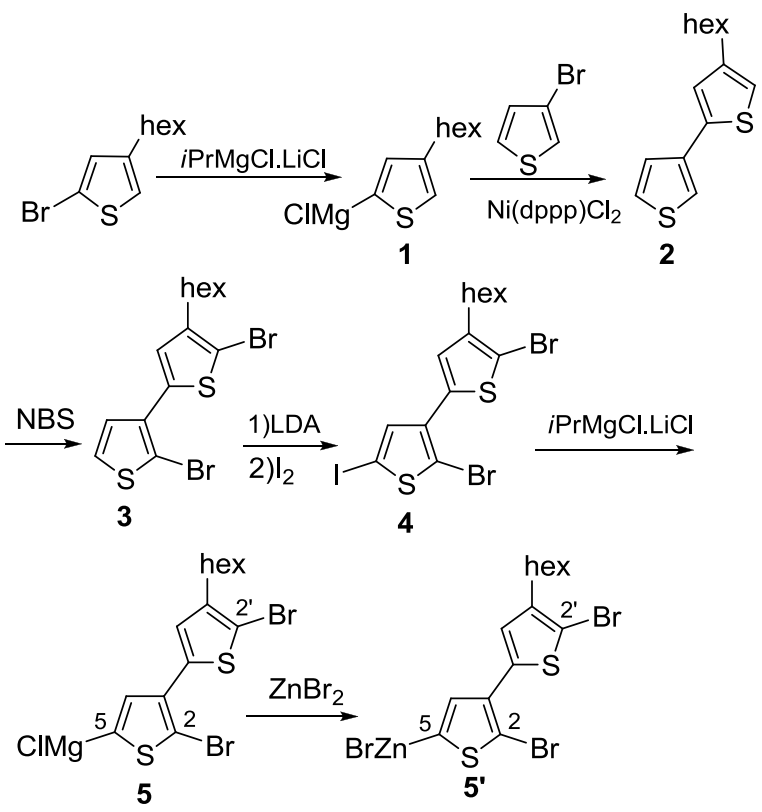

Scheme 1. Synthesis of the branched bithiophene monomer 5.

The degree of nonlinearity, for the polymers prepared by this monomer, is defined as the amount of polymerizations in 2',5-fashion compared to the sum of polymerizations in 2',5 and 2,5-fashion (Scheme 2). 


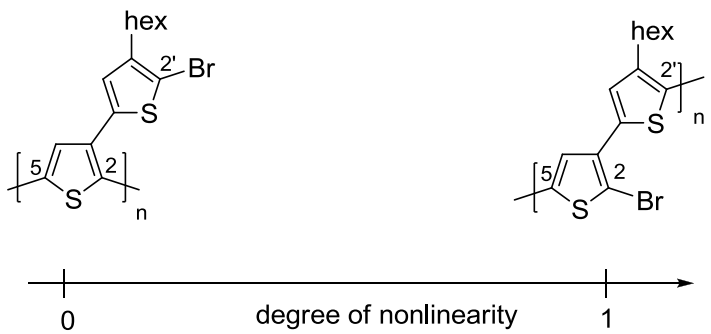

Scheme 2. Change in polymer structure going from completely linear to complete nonlinear polymers.

A first attempt towards control over the amount of branching is the polymerization of the branched monomer $\mathbf{5 / 5}$, with three different external initiators, more specifically ArNi(dppp)Br $(\mathbf{8})^{38}, \operatorname{ArNi}\left(\mathrm{PPh}_{3}\right)_{2} \mathrm{Br}^{39}(\mathbf{9})$ and $\operatorname{ArPd}(\mathrm{RuPhos}) \mathrm{Br}^{40}(\mathbf{1 0})$, leading to polymers P1-100, P2-100 and P3-100, respectively (Scheme 3).
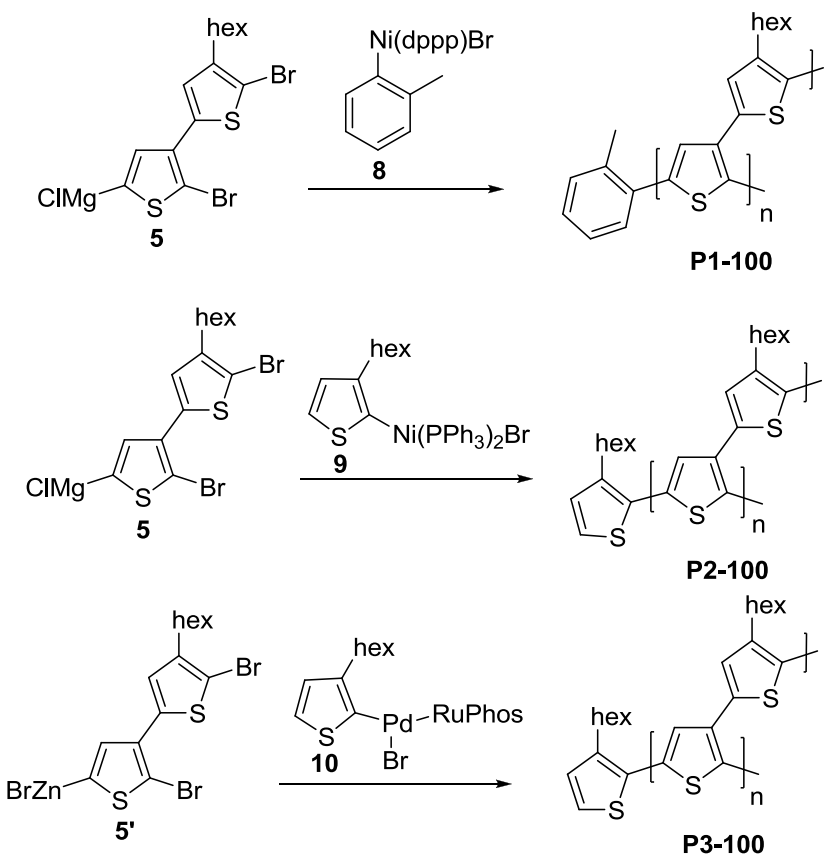

Scheme 3. Synthesis of $\mathbf{P 1 / 2 / 3 - 1 0 0}$ by polymerization of the branched monomer $\mathbf{5 / 5}$, with three different catalysts.

Two different polymerization protocols are employed. The first one is a Kumada catalysttransfer polycondensation (KCTP). ${ }^{41}$ For this protocol two different ligands are employed. 
When dppp is used as ligand the Ni-catalyst remains complexed to the $\pi$-system of the polymer backbone. In case of linear poly(3-hexylthiophene), this prevents transfer and termination reactions. ${ }^{42,43}$ Although a polycondensation, the polymerization proceeds in a chain growth manner due to this complexation. ${ }^{44}$ While complexed to the backbone, the catalyst can walk back and forth along the chain (ring walking). The only way branching can occur, is by walking backwards and insert in the $\mathrm{C}-\mathrm{Br}$ bond of a thiophene side chain. Nonlinear growth can also occur by oxidative insertion in the $\mathrm{C}-\mathrm{Br}$ of a 3'-hexylthienyl sidechain of a terminal unit. Concerning nonlinearity, due to the higher inaccessibility of the $\mathrm{C}-\mathrm{Br}$ bond of the thiophene side chain compared to $\mathrm{C}-\mathrm{Br}$ bond of the linear backbone, linear growth will be more probable as nonlinear growth.

The other ligand used is $\mathrm{PPh}_{3}$. The Ni-catalyst with $\mathrm{PPh}_{3}$ is less strongly complexed to the $\pi$ backbone. As a consequence, there is a probability that the catalyst dissociates and then reinserts in a $\mathrm{C}-\mathrm{Br}$ bond of the linear chain or in the $\mathrm{C}-\mathrm{Br}$ bond of the thiophene side chains. Compared to $\mathrm{Ni}(\mathrm{dppp})$, branching cannot only occur by ringwalking, but also by reinsertion. However, $\mathrm{Ni}\left(\mathrm{PPh}_{3}\right)_{2}$ being rather unstable in solution, there is high probability of premature termination before reinsertion occurs, limiting the presence of branches. ${ }^{45,46}$ Nonlinearity can occur in the same way as in the previous case, but also by reinsertion in a terminal unit.

The second protocol to polymerize monomer 5', is the $\mathrm{Pd}(\mathrm{RuPhos})$ protocol, using Negishi couplings. ${ }^{40}$ Compared to the Ni-catalysts used in the KCTP protocol, the $\mathrm{Pd}(\mathrm{RuPhos})$-catalyst has a small affinity for the polymer backbone. After the coupling reaction it dissociates. Branching occurs by reinsertion in a dangling $\mathrm{C}-\mathrm{Br}$ bond. As the $\mathrm{C}-\mathrm{Br}$ bonds in the 3'hexylthienyl side chains are equally reactive as the $\mathrm{C}-\mathrm{Br}$ bond at end of the linear backbone, the highest degree of nonlinearity is expected for the polymer made by the $\mathrm{Pd}(\mathrm{RuPhos})$ protocol. 
A second approach to achieve control over the degree of nonlinearity is by copolymerizing the branched and a linear monomer in different ratios. A series of 5 polymers were synthesized via KCTP with a percentage of branched monomer of $0,5,10,25$ and $100 \%$ corresponding with P1-0, P1-5, P1-10, P1-25 and P1-100 respectively (Scheme 4). We aimed for a degree of polymerization of 20 by adjusting the initiator to monomer ratio.

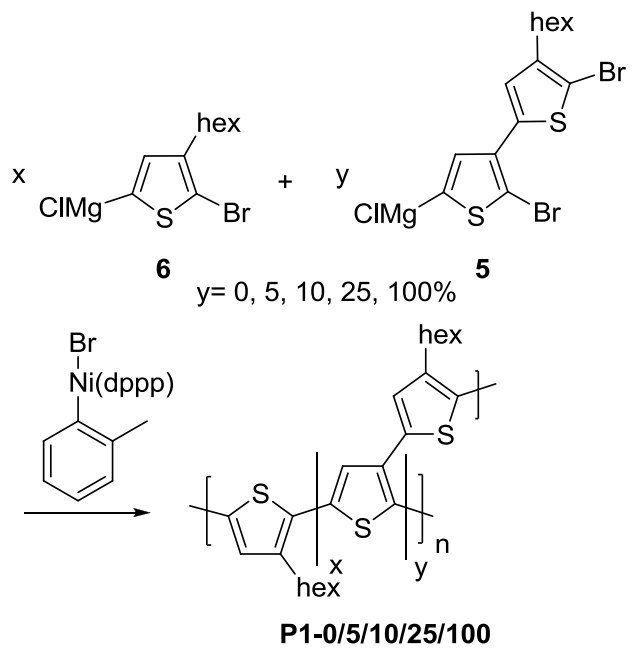

Scheme 4. Copolymerization of a linear and branched thiophene monomer in different ratios via KCTP.

\section{GPC}

In order to have an idea of the molar mass and the dispersity, GPC spectra were recorded. ${ }^{47,48}$ In Table 1, the number-average molar masses, determined against polystyrene standards, and $Ð$ are listed. For the branched polymers prepared via three different catalysts we measure a higher Đ for P2-100 $\left(\mathrm{Ni}\left(\mathrm{PPh}_{3}\right)_{2}\right)$ (Table 1). This can be explained due to a loss of the controlled nature of the polymerization. ${ }^{49}$ The number-average molar masses are quite similar.

For the polymers synthesized via the second approach $(\mathbf{P 1 - 0 / 5 / 1 0 / 2 5 / 1 0 0})$ the molar masses and $Đ$ are in the same range (Table 2). Therefore, the only main changing parameter for the different polymers is their degree of nonlinearity. The low $Đ$ values are a good indication that 
the controlled nature of the polymerization is conserved when polymerizing a branched instead of a linear monomer.

Table 1. GPC data for the series of the branched poly(thiophenes) and determination of the degree of nonlinearity via ${ }^{1} \mathrm{H}-\mathrm{NMR}$ (see further). a Determined according to formula $\frac{3 b}{2 d} \quad \mathrm{~b}$ Determined by multiplying incorporation 5 (\%) x nonlinearity (\%) of P1-100

\begin{tabular}{cccccc}
\hline Polymer & $\bar{M}_{\mathrm{n}}(\mathrm{kg} / \mathrm{mol})$ & $\begin{array}{c}\text { Incorporation 5 } \\
(\%)\left({ }^{1} \mathrm{H}-\mathrm{NMR}\right)\end{array}$ & $\begin{array}{c}\text { Nonlinearity (\%) } \\
\left({ }^{1} \mathrm{H}-\mathrm{NMR}\right)^{\mathrm{a}}\end{array}$ & $\begin{array}{c}\text { Nonlinearity (\%) } \\
\text { expected }^{\mathrm{b}}\end{array}$ \\
\hline P1-0 & 4.1 & 1.2 & 0 & 0 & 0 \\
\hline P1-5 & 4.1 & 1.2 & 6 & 2 & 2 \\
\hline P1-10 & 4.5 & 1.2 & 9 & 8 & 4 \\
\hline P1-25 & 4.6 & 1.3 & 23 & 12 & 9 \\
\hline P1-100 & 4.3 & 1.3 & 100 & 41 & $/$ \\
\hline P2-100 & 4.8 & 1.9 & 100 & 42 & $/$ \\
\hline P3-100 & 3.7 & 1.4 & 100 & 62 & \\
\hline
\end{tabular}

\section{${ }^{1}$ H-NMR spectroscopy}

${ }^{1} \mathrm{H}-\mathrm{NMR}$ analysis was performed to determine the incorporation of the branched monomer in the second series and the degree of nonlinearity for both series of polymers. To determine the degree of nonlinearity of the first series (P1/2/3-100) the peaks corresponding to the $\alpha-\mathrm{CH}_{2}$ of the hexyl side chain were analyzed and integrated. Two distinct peaks can be assigned for the $\alpha-\mathrm{CH}_{2}$ protons: one corresponds to a monomer unit that did not grow further via the thiophene side chain i.e. in a linear fashion (a). The other peak (b) corresponds with a monomer unit that grew further at the thiophene side chain (Figure 1). 


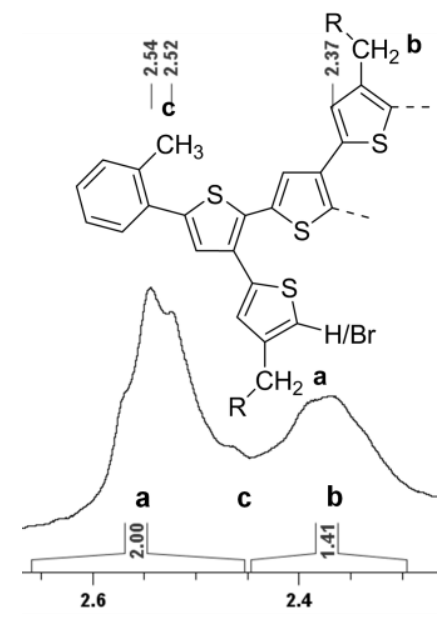

Figure 1. Peaks corresponding with the $\alpha-\mathrm{CH}_{2}$ of the hexyl side chain of P1-100.

By integrating those peaks the degree of nonlinearity can be calculated by approximation from following formula:

$$
\text { degree of nonlinearity }=\frac{b}{a+b}
$$

This is only an approximation as the $o$-tolyl peak of the initiator overlaps around $2.5 \mathrm{ppm}$. The results for polymers $\mathbf{P 1 / 2 / 3 - 1 0 0}$ can be found in Table 1 . There is a clear difference in the degree of nonlinearity for the polymers prepared via the $\mathrm{Pd}(\mathrm{RuPhos})$-catalyst (62\%) (P3-100) compared to the polymers made with the Ni-catalysts $(\mathbf{P 1 / 2 - 1 0 0})$. This can be explained as postulated before. Calculation of the degree of branching is beyond the scope of the current techniques as there is scarcely a difference in ppm value for the $\alpha-\mathrm{CH}_{2}$ of the hexyl side chain of a unit grown in a nonlinear way compared to the $\alpha-\mathrm{CH}_{2}$ of the hexyl side chain of a unit at a branching point.

Note that the calculated degree of nonlinearity, based on ${ }^{1} \mathrm{H}-\mathrm{NMR}$, for P1-100 differs from the result obtained by the group of Mori for a polymerization of a branched terthiophene with a similar catalyst, $\mathrm{Ni}(\mathrm{dppp}) \mathrm{Cl}_{2} \cdot{ }^{29}$ Based on viscosity measurements and ${ }^{1} \mathrm{H}-\mathrm{NMR}$ they state that the degree of nonlinearity is 0 and as a consequence they conclude the polymerization proceeds in a linear fashion. The only possible explanation for this is the difference in monomer structure. 
The ratio of incorporation of the branched monomer in the copolymer series $(\mathbf{P 1 - 0 / 5 / 1 0 / 2 5 / 1 0 0})$ was determined by ${ }^{1} \mathrm{H}-\mathrm{NMR}$ spectroscopy as well. The integration values for the peak of the $\alpha-\mathrm{CH}_{2}$ of the hexyl side chain of a linear unit (e) and of the overlapping peaks of the terminal $\mathrm{CH}_{3}$ groups of the linear and branched units (d) (Figure 2) were determined.

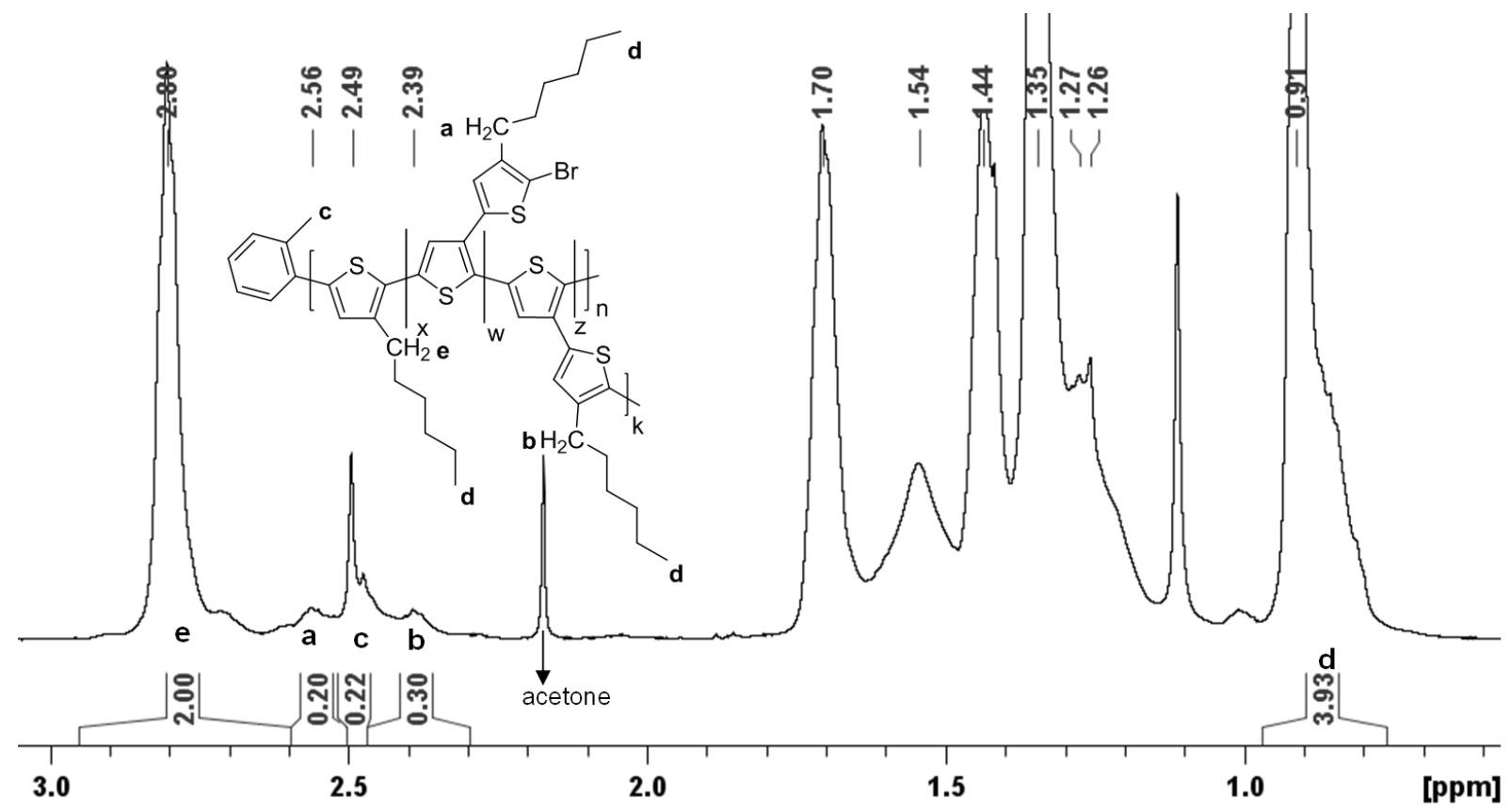

Figure 2. ${ }^{1} \mathrm{H}-\mathrm{NMR}$ integration of the signals of $\alpha-\mathrm{CH}_{2}$ protons of the hexyl side chain of the linear (e) and branched units (a/b) and the terminal $\mathrm{CH}_{3}$ protons (d) of the hexyl chain of linear and branched units of P1-25.

These values were then introduced in the following formula:

$$
\text { degree branched units incorporated }=1-\frac{3 e}{2 d}
$$

We can conclude that the ratio of branched monomer to linear monomers incorporated in the polymer corresponds very well with the ratio of the monomers used in the polymerization mixture (Table 1). This can be explained by their equal reactivity.

Also for the polymer series $(\mathbf{P 1 - 0 / 5 / 1 0 / 2 5 / 1 0 0})$ the degree of nonlinearity could be calculated using the integration values of the ${ }^{1} \mathrm{H}-\mathrm{NMR}$ peaks. Protons $\mathrm{b}$ correspond with the $\alpha-\mathrm{CH}_{2}$ of a 
branched monomer unit elongated at the 3'-hexylthienyl side chain (nonlinear growth). Integration of protons d corresponds with the terminal $-\mathrm{CH}_{3}$ groups of the hexyl side chain of the linear and branched monomers (Figure 2).

Consequently, following formula gives the degree of nonlinearity:

$$
\text { degree of nonlinearity }=\frac{3 b}{2 d}
$$

The calculated values are close to the expected values, which can be calculated as follows. Analysis of P1-100 indicated there is 41 percent chance of nonlinear growth. By multiplying the chance of nonlinear growth with the degree of incorporated branched units the expected degree of nonlinearity can be estimated. These estimated values are close to the experimental values determined via ${ }^{1} \mathrm{H}-\mathrm{NMR}$ (Table 1$)$.

\section{UV-vis spectra}

To investigate the self-assembly in solution, $\mathrm{UV}$-vis spectra were recorded in pure $\mathrm{CHCl}_{3}$ and a $\mathrm{MeOH} / \mathrm{CHCl}_{3}(90 / 10)$ mixture. For the series $\mathbf{P 1 / 2 / 3 - 1 0 0}$ we can clearly distinguish two different peaks (Figure 3) in the spectra: one originating from the $\alpha-\alpha$ conjugation around 450 $\mathrm{nm}$ and the other corresponding with an $\alpha-\beta$ conjugation state around $300 \mathrm{~nm} .^{30}$

For the spectra in the good solvent $\left(\mathrm{CHCl}_{3}\right)$ the trend in $\lambda_{\text {max,abs }}$ can easily be explained. As the amount of $\alpha-\alpha$ conjugation decreases with increasing nonlinearity, the observed trend in $\lambda_{\text {max,abs }}$ (around $450 \mathrm{~nm}$ ) is perfectly in line with the increasing degree of nonlinearity $(\mathbf{P 1 - 1 0 0} \rightarrow$ P3100). Indeed, shorter linear chain segments lead to a blueshift in the $\lambda_{\text {max,abs. }}$

In nonsolvent $(90 \% \mathrm{MeOH})$ the $\lambda_{\text {max,abs }}$ values do not shift compared to the spectra in good solvent. The absence of a redshift is attributed to the presence of branches which complicates the planarization (Table 2). Only for P1-100 there is a shoulder visible around $580 \mathrm{~nm}$. This vibrational fine structure means there is some long-range order in polymer P1-100. This is not 
visible for P2-100 and P3-100 due to their higher degree of nonlinearity, complicating the formation of aggregates and long range order.
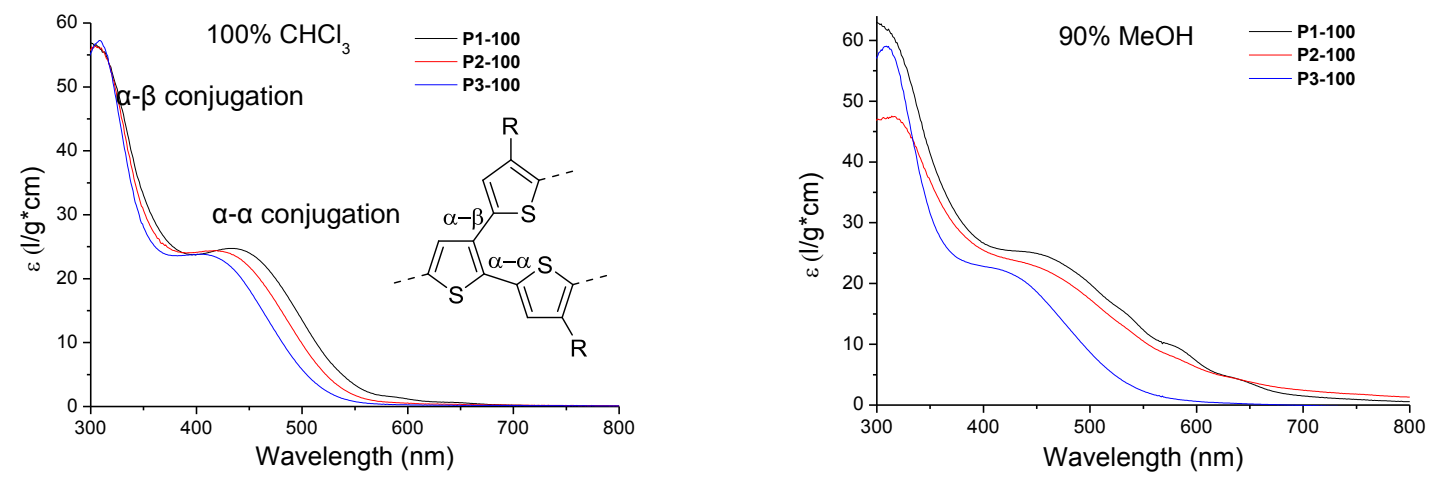

Figure 3. The UV-vis spectra in good solvent $\left(\mathrm{CHCl}_{3}\right)$ and nonsolvent $\left(\mathrm{MeOH} / \mathrm{CHCl}_{3}(90 / 10)\right)$ for series (P1/2/3-100) of branched poly(thiophenes).

For the series $(\mathbf{P 1 - 0 / 5 / 1 0 / 2 5 / 1 0 0 )}$ an increase of the peak around $300 \mathrm{~nm}$ for increasing percentages of branched monomer incorporated in the polymer (Figure 4) is observed, as only in the branched monomer units $\alpha-\beta$ conjugation segments are present.

In good solvent $\left(\mathrm{CHCl}_{3}\right)$ the $\lambda_{\text {max,abs }}$ values show a blueshift for increasing amounts of branched monomer, incorporated, except for P1-100. This trend is explained by the shortening of the conjugation length by incorporating a branched monomer. Other properties, unknown to us, must account for the different behavior of P1-100.

When comparing the spectra in good solvent $\left(\mathrm{CHCl}_{3}\right)$ and a poor solvent mixture $(90 \% \mathrm{MeOH})$, the redshift in general decreases with higher degree of incorporation of the branched monomer as branching compromises the planarization and stacking of the polymer chains (Table 2). ${ }^{30}$ However the highest redshift is observed for P1-5 and not for P1-0. This is related to a phenomenon, observed by our group and others, that the introduction of molecular disorder can improve the stacking properties of the material..$^{50-53}$ The absence or presence of fine structure (shoulder around $580 \mathrm{~nm}$ ) in the $90 \% \mathrm{MeOH}$ spectra can be understood in terms of the 
uniformity of the side chain and concomitant ease/difficulty to order. Homopolymers P1-100 and P1-0 show a clear shoulder. Linear poly(3-hexylthiophene) (P1-0) with its aliphatic hexyl sidechain, is known to stack very well. Homopolymer P1-100 can be considered as a linear polymer with all hexyl side chains replaced by alkylthienyl side chains. For the copolymers, from P1-5 $\rightarrow$ P1-25, due to the increasing implementation of aromatic 3'-hexylthienyl side chains instead of the aliphatic hexyl side chain, the uniformity in the nature of the side chains decreases and, as a consequence, the disorder in the polymer chain increases. For P1-5 there is still a shoulder present, because the content of aromatic 3'-hexylthienyl side chains is still very low. For P1-10 only the onset of the shoulder is visible, while for P1-25 all fine structure is lost due to its very low uniformity in the nature of the side chain. This loss of fine structure means that long-range order in the aggregates is absent.
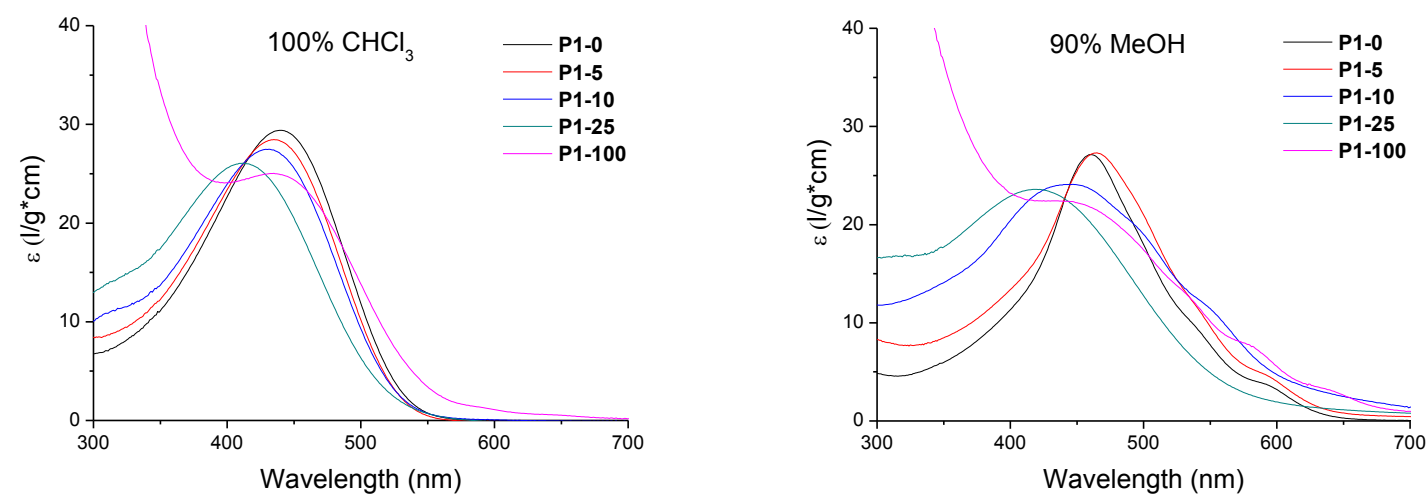

Figure 4. The UV-vis spectra in pure $\mathrm{CHCl}_{3}$ and $\mathrm{MeOH} / \mathrm{CHCl}_{3}(90 / 10)$ for series (P1$\mathbf{0} / \mathbf{5} / \mathbf{1 0} / \mathbf{2 5} / \mathbf{1 0 0})$ of poly(thiophene) copolymers. 
Table 2. Optical data for polymer series (P1/2/3-100) of branched poly(thiophenes) and for the copolymer series $(\mathbf{P 1 - 0 / 5 / 1 0 / 2 5 / 1 0 0 )}$.

\begin{tabular}{|c|c|c|c|c|}
\hline Polymer & $\begin{array}{c}\lambda_{\max , \mathrm{abs}} \\
\left(\mathrm{CHCl}_{3}\right) \\
(\mathrm{nm})\end{array}$ & $\begin{array}{c}\lambda_{\max , \mathrm{abs}} \\
(\mathrm{MeOH}) \\
(\mathrm{nm})\end{array}$ & $\Delta \lambda(\mathrm{nm})$ & $\begin{array}{c}\lambda_{\text {max }, \mathrm{em}} \\
\left(\mathrm{CHCl}_{3}\right) \\
(\mathrm{nm})\end{array}$ \\
\hline P1-0 & 439 & 461 & 22 & 573 \\
\hline P1-5 & 435 & 465 & 30 & 573 \\
\hline P1-10 & 431 & 447 & 16 & 569 \\
\hline P1-25 & 412 & 419 & 7 & 566 \\
\hline P1-100 & 432 & 432 & 0 & 586 \\
\hline P2-100 & 424 & 424 & 0 & 581 \\
\hline P3-100 & 405 & 405 & 0 & 574 \\
\hline
\end{tabular}

\section{Fluorescence}

Emission spectra were recorded in pure $\mathrm{CHCl}_{3}$ and $90 \% \mathrm{MeOH}$ by excitation at $400 \mathrm{~nm}$. For the first series $(\mathbf{P 1 / 2 / 3 - 1 0 0})$ in pure $\mathrm{CHCl}_{3}$ the $\lambda_{\text {max,em }}$ (Table 2) decreases in the order of $\mathrm{Ni}(\mathrm{dppp}), \mathrm{Ni}\left(\mathrm{PPh}_{3}\right)_{2}, \mathrm{Pd}(\mathrm{RuPhos})$-catalyst (Figure 5). This can be explained due to the fact that emission spectra follow in good accordance the trend of longest $\alpha$ - $\alpha$-conjugation. ${ }^{30}$

For the spectra in a poor solvent mixture $(90 \% \mathrm{MeOH})$ the intensity drops by a factor $10-50$ compared to spectra in good solvent $\left(\mathrm{CHCl}_{3}\right)$. Only for the polymer synthesized with the $\operatorname{Pd}(\mathrm{RuPhos})$-catalyst (P3-100) there is still a peak visible that corresponds to residual fluorescence of non-aggregated polymer chains. This observation is explained by difference in degree of nonlinearity. A higher degree of nonlinearity and therefore disorder complicates the formation of aggregates. 

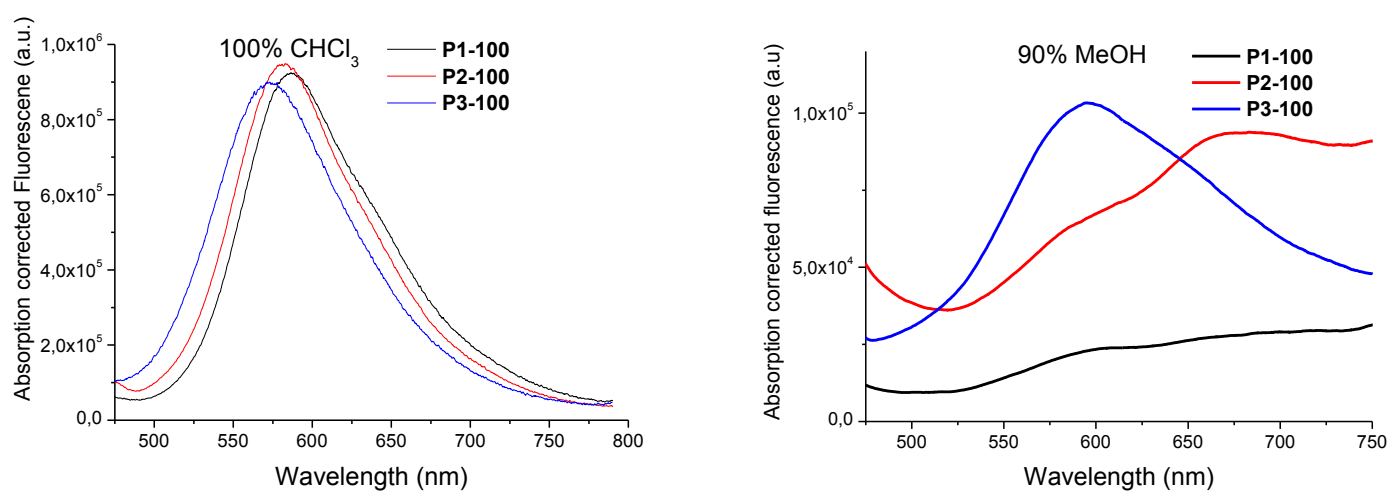

Figure 5. Fluorescence spectra of polymer series $(\mathbf{P 1 / 2 / 3 - 1 0 0})$ in pure $\mathrm{CHCl}_{3}$ and $\mathrm{MeOH} / \mathrm{CHCl}_{3}(90 / 10)$.

For the fluorescence spectra of the copolymer series $(\mathbf{P 1 - 0 / 5 / 1 0 / 2 5 / 1 0 0})$ in pure $\mathrm{CHCl}_{3}$ the intensity for P1-25 and P1-100 (Figure 6) is clearly lower than for the rest. The intensity for P1-10 is also slightly lower as for P1-5 and P1-0. As we excite at $400 \mathrm{~nm}$, electronic states of $\alpha-\beta$ conjugation can be excited. In a next step the absorbed energy will be transferred to the longest chromophoric $\alpha-\alpha$ conjugation before photoemission takes place. ${ }^{28}$ Energy loss during transfer may account for the drop in intensity. The blueshift for higher degrees of nonlinearity is explained by the fact that photoluminescence comes mainly from the longest chromophoric $\alpha$ - $\alpha$-conjugation, shortening with increasing nonlinearity. The reason why P1-100 does not follow this trend is not clear.

For the spectra in a poor solvent mixture $(90 \% \mathrm{MeOH})$ there is a drop of intensity by a factor $500(\mathbf{P 1 - 0})$ to $50(\mathbf{P 1 - 1 0 0})$ compared to the spectra in pure $\mathrm{CHCl}_{3}$. The amount of decrease in intensity is related to the tendency of planarization of the polymer chains. More linear polymers will tend to form aggregates of closely stacked planar chains causing less relaxation by photoemission. The observed spectra are a result of the intrinsic fluorescence in good solvent and the residual fluorescence of non-aggregated chains. 

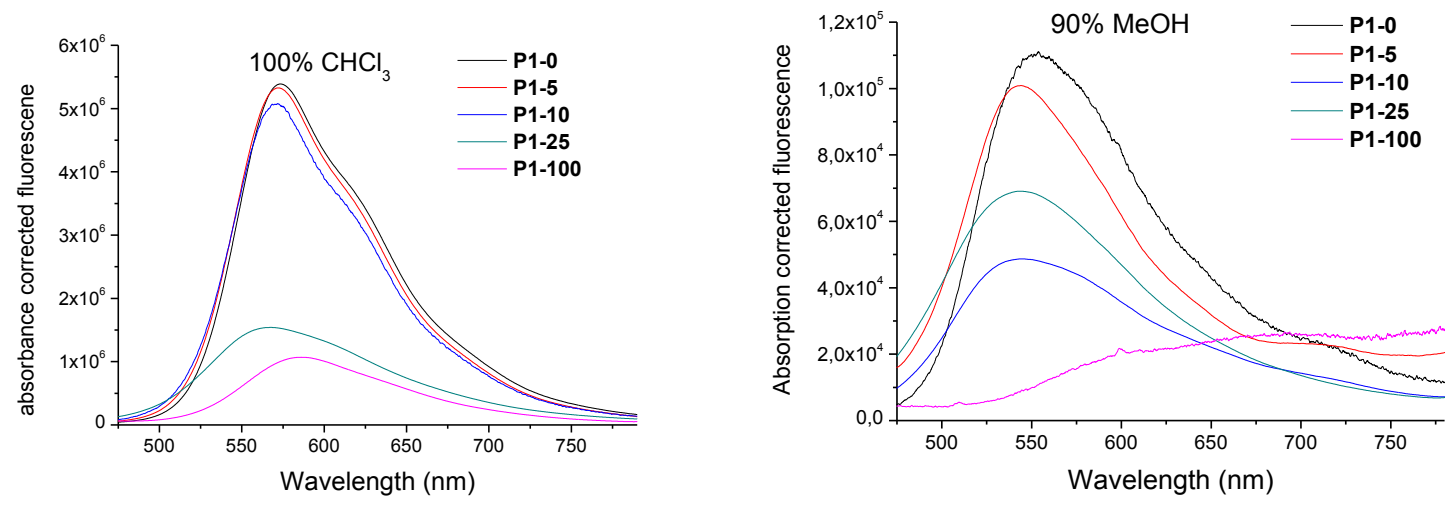

Figure 6. Fluorescence spectra of series $(\mathbf{P 1 - 1 0 0 / 2 5 / 1 0 / 5 / 0})$ of poly(thiophene) copolymers in pure $\mathrm{CHCl}_{3}$ and $90 \% \mathrm{MeOH}$.

\section{DSC}

The properties in solid state of the materials of the copolymer series $(\mathbf{P 1 - 0 / 5 / 1 0 / 2 5 / 1 0 0})$ were studied by DSC and AFM. DSC-spectra (see SI) were recorded to study the thermal properties of the materials. Only for polymers P1-0/5/10 a crystallization peak was observed. There is a decrease in crystallization temperature for increasing nonlinearity degree (Table 3). This is in agreement with the fact that more linear polymers form aggregates with stronger intermolecular interactions. 
Table 3. Crystallization temperatures for poly(thiophene) copolymer series $(\mathbf{P 1 - 0 / 5 / 1 0 / 2 5 / 1 0 0})$ from DSC.

\begin{tabular}{cc}
\hline Polymer & $\mathrm{T}_{\mathrm{c}}$ \\
P1-0 & 136 \\
P1-5 & 126 \\
P1-10 & 107 \\
P1-25 & $/$ \\
\hline P1-100 & $/$
\end{tabular}

\section{AFM}

More information on the morphology and the electrical properties of the materials of the series (P1-0/5/10/25/100) was obtained by AFM measurements of thin film samples. These samples were prepared from either $o$-xylene or THF solutions. It appeared that the nature of the solvent is not influencing the observed supramolecular morphologies of the conjugated polymers. The thin deposits $(25 \mu 1)$ on glass substrates are then allowed to dry overnight in a closed chamber containing the corresponding solvent. By this solvent annealing process, the polymer chains can interact to reach the most thermodynamically stable organization

Figure 7 illustrates the obtained morphologies for $0,5,25$ and $100 \%$ branched system. On the Figure $7 \mathrm{a}$, typical P3HT fibrils are obtained by $\pi-\pi$ interactions. As already described in literature the conjugated P3HT backbones are stacked together to form nanofibrils with a length of few micrometers and a width of 20-25 nm corresponding to the length of the chains. When the branching percentage is increased (Figures $7 b$ and c), more lateral connections are now possible due to the topology of the polymer chains. For the $100 \%$ branched system, no fibrils are observed and the thin film seem to be amorphous. From this morphological characterization, 
we can conclude that the branched percentage is strongly influencing the supramolecular organization depending on the fact that the stacking of the conjugated P3HT backbones are allowed to occur or not.
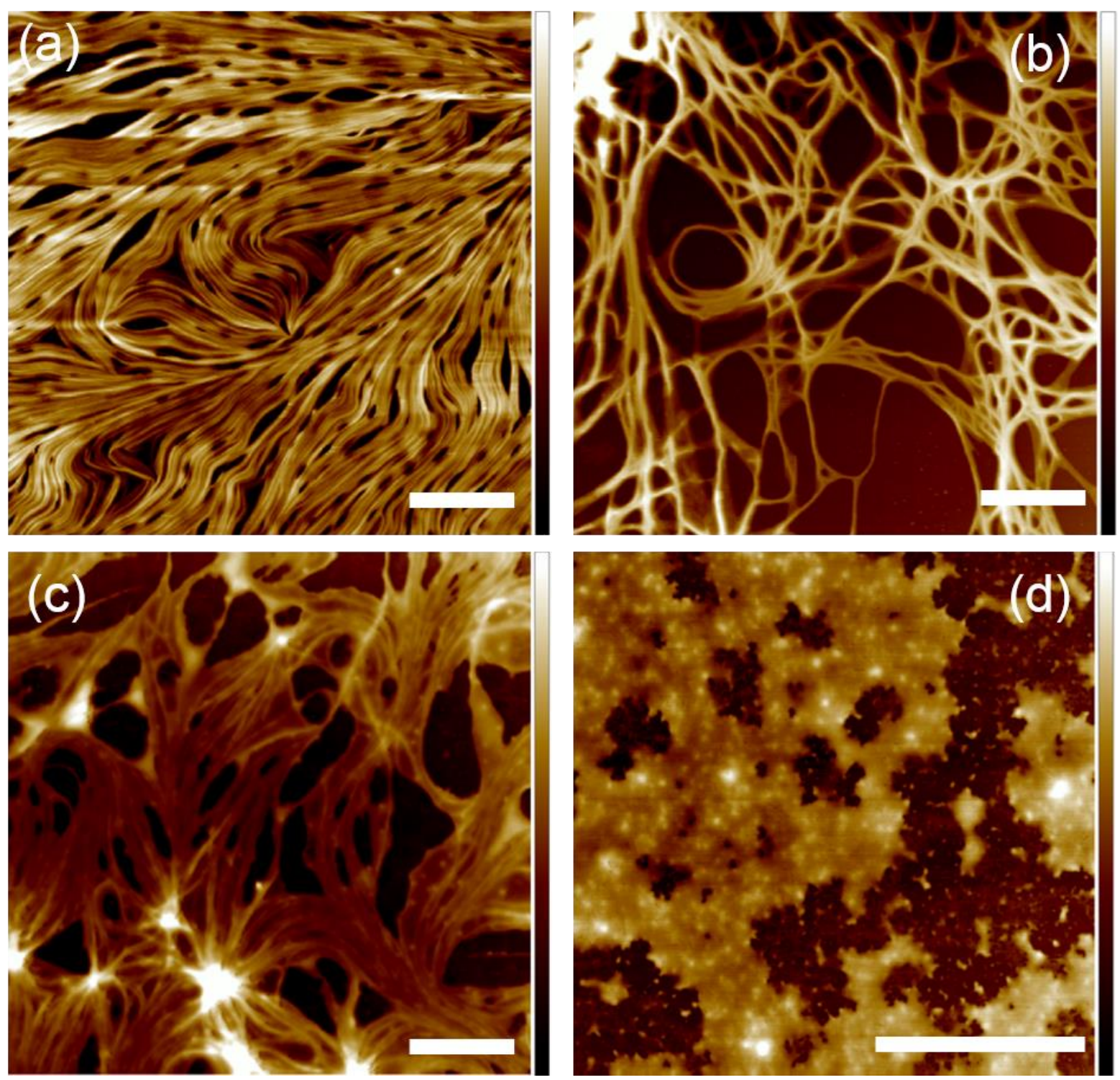

Figure 7. Height Tapping Mode images of branched P3HT thin deposit from $1 \mathrm{mg} / \mathrm{mL} o$ Xylene solution: (a) $0 \%$, (b) 5\%; (c) 25\%, (d) 100\%. The scale bar corresponds to $2 \mu \mathrm{m}$ and the Z-range bar is $40 \mathrm{~nm}$.

For the electrical characterization of the samples, Conductive AFM (CAFM) was performed in contact mode. Figures 8 a and c show the results obtained on the 0 and $25 \%$ of branched chains. 
The light brown colored areas on the Figure $8 \mathrm{~b}$ and $\mathrm{d}$ correspond to zero-current zones. As expected, when a DC bias of $-2 \mathrm{~V}$ is applied between the tip and the sample, the fibrillar $\mathrm{P} 3 \mathrm{HT}$ appears with a negative current (dark brown lines on Figure 8 b) for all the fibrils while for the $25 \%$, only few fibrils are visible in the current images; For the corresponding topographic image (Figure $8 \mathrm{c}$ ), these fibrils are not distinguishable. This means that for branched P3HT materials, the current can flow only when the conjugated segments are perfectly packed and electrical connection are not possible between P3HT fibrils.

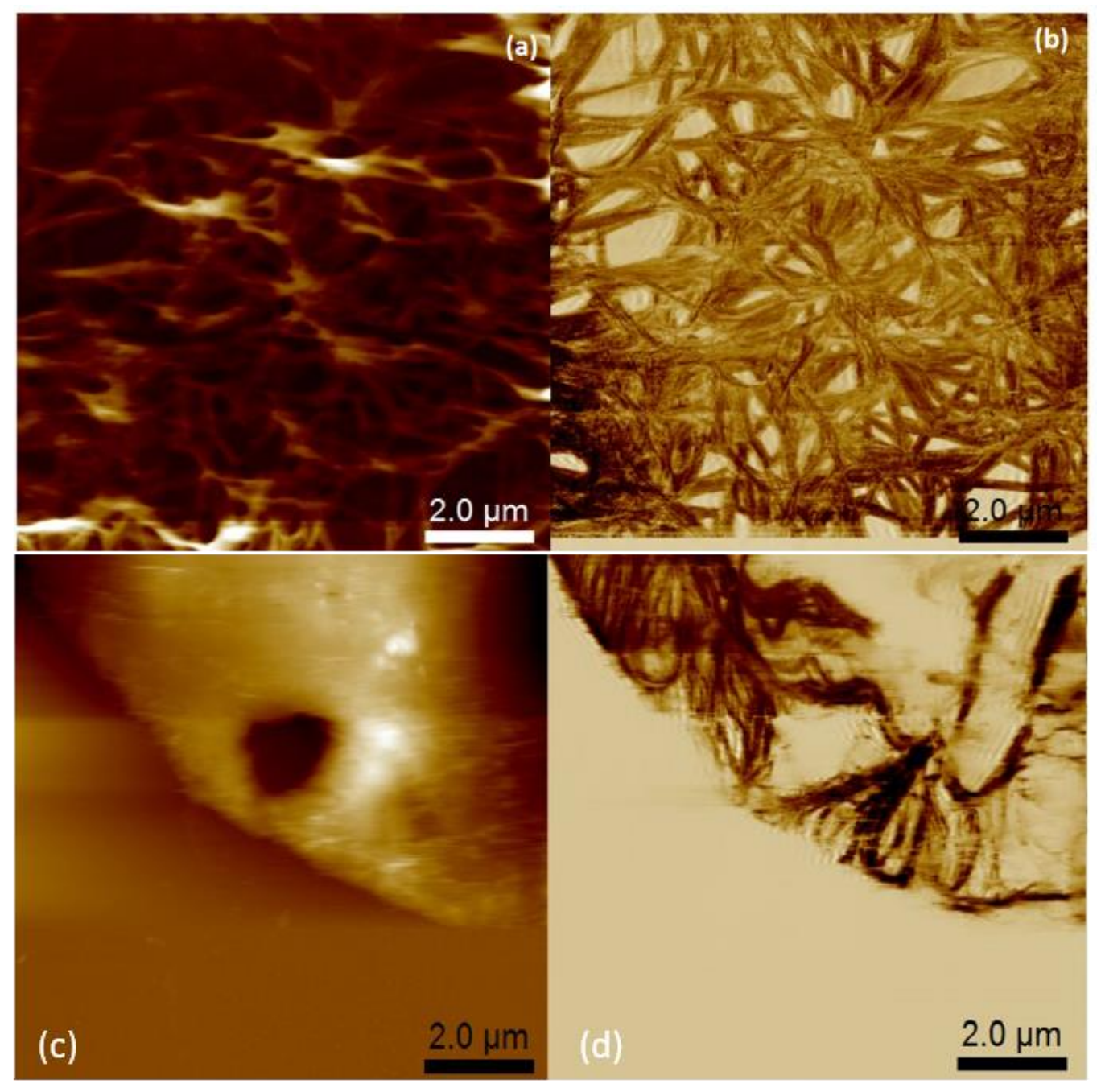

Figure 8. Topographic and current Conductive AFM images of branched P3HT thin deposit from $1 \mathrm{mg} / \mathrm{mL} o$-Xylene solution (a) Topographic and (b) current image of 0\%; (c) topographic 
and (d) current image of $25 \%$. The Z-range is $100 \mathrm{~nm}$. The current range is $20 \mathrm{pA}$ for (b) and $60 \mathrm{pA}$ for $(\mathrm{d})$.

\section{CONCLUSION}

Two different routes towards control of the nonlinearity of poly(thiophenes) were investigated. It was proven by ${ }^{1} \mathrm{H}-\mathrm{NMR}$ that the degree of nonlinearity can be controlled using a different catalyst or by copolymerizing a linear and branched monomer in different ratios. A maximum degree of nonlinearity of $62 \%$ was achieved via the homopolymerization of a branched bithiophene monomer using the $\operatorname{Pd}(\mathrm{RuPhos})$-catalyst. For lower degrees of nonlinearity the percentage of nonlinearity could be varied on a continuous scale by copolymerizing a branched and linear monomer. The influence of the nonlinearity degree on the optoelectronic properties, related to the macro- and supramolecular structures, was studied by UV-vis and fluorescence spectroscopy. Analysis of the spectra revealed that two aspects determine the supramolecular organization and optical properties: first, the degree of nonlinearity which is related to the length of the $\alpha-\alpha$ conjugated segments: nonlinearity increase the bandgap and complicates a good stacking. Second, for the copolymer series made of a branched and linear monomer also the uniformity in the nature of the side chain of the polymer backbone affects the spectra: a good supramolecular organization is inhibited in the copolymers, as this results in no uniform side-chains.. The crystallinity and morphology of the materials were studied via AFM. Severe nonlinearity results in the disappearance of the crystalline fibrillary structure. The introduction of branching results in lateral connections between the fibrils, but the average conductivity is lower with the increase of the branched percentage. The electrical connection between the conjugated branches is not observed by CAFM.

\section{ASSOCIATED CONTENT}


Supporting Information. Synthesis of the branched monomer, homopolymers (P1/2/3-100) and copolymers (P1-0/5/10/25), ${ }^{1} \mathrm{H}-\mathrm{NMR}$-spectra of the homo polymers (P1/2/3-100) and the copolymers (P1-0/5/10/25), the DSC thermograms of P1-100/25/10/5/0. This material is available free of charge via the Internet at http://pubs.acs.org.

\section{AUTHOR INFORMATION}

\section{Corresponding Authors}

*(G.K.) E-mail: guy.koeckelberghs@chem.kuleuven.be.

*(Ph.L.) E-mail: philippe.leclere@umons.ac.be

\section{Author Contributions}

The manuscript was written through contributions of all authors. All authors have given approval to the final version of the manuscript.

Funding Sources

Onderzoeksfonds KU Leuven/Research Fund KU Leuven, Fund for Scientific Research (FWOVlaanderen), Science Policy Office of the Belgian Federal Government (PAI 7/5), the European Commission/Région Wallonne FEDER program and "Fonds National pour la Recherche Scientifique" (FRS-FNRS). JS is an IWT doctoral fellow. Ph.L. is F.R.S.-FNRS Senior Research Associate.

\section{ACKNOWLEDGMENTS}

We are grateful to the Onderzoeksfonds KU Leuven/Research Fund KU Leuven and the Fund for Scientific Research (FWO-Vlaanderen) for financial support. JS is grateful to IWT for a doctoral fellowship. Research in Mons is supported by the Science Policy Office of the Belgian 
Federal Government (PAI 7/5), the European Commission/Région Wallonne FEDER program, and the "Fonds National pour la Recherche Scientifique" (FRS-FNRS). Ph.L. is F.R.S.-FNRS Senior Research Associate.

\section{ABBREVIATIONS}

AFM (Atomic Force Microscopy); CAFM (Conductive Atomic Force Microscopy), DSC (differential scanning calorimetry); GPC (gel permeation chromatography); GRIM (Grignard Metathesis); KTCP (Kumada Catalyst Transfer Polymerization); LDA (lithium diisopropylamide); $\bar{M}_{n}$ (number average molar mass); NBS ( $N$-bromosuccinimide); DP (Degree of Polymerization)

\section{REFERENCES}

(1) Steverlynck, J.; De Winter, J.; Gerbaux, P.; Lazzaroni, R.; Leclère, P.; Koeckelberghs, G. Influence of the Grafting Density on the Self-Assembly in Poly(phenyleneethynylene)-g-poly(3-Hexylthiophene) Graft Copolymers. Macromolecules 2015, 48 (24), 8789-8796

(2) Steverlynck, J.; De Cattelle, A.; De Winter, J.; Gerbaux, P.; Koeckelberghs, G. Energy Transfer in poly(3-Hexylthiophene)-g-Polyfluorene Graft Copolymers. J. Polym. Sci. Part A Polym. Chem. 2016, 54 (9), 1252-1258

(3) Zeigler, D. F.; Mazzio, K. A.; Luscombe, C. K. Fully Conjugated Graft Copolymers Comprising a P-Type Donor-Acceptor Backbone and Poly(3-Hexylthiophene) Side Chains Synthesized Via a "Graft Through" Approach. Macromolecules 2014, 47 (15), 5019-5028

(4) Wang, J.; Lu, C.; Mizobe, T.; Ueda, M.; Chen, W.-C.; Higashihara, T. Synthesis and Characterization of All-Conjugated Graft Copolymers Comprised of N-Type or P-Type Backbones and Poly(3-Hexylthiophene) Side Chains. Macromolecules 2013, 46 (5), 1783-1793

(5) Kalow, J. A.; Swager, T. M. Synthesis of Miktoarm Branched Conjugated Copolymers by ROMPing In and Out. ACS Macro Lett. 2015, 4 (11), 1229-1233

(6) Jarosz, T.; Lapkowski, M.; Ledwon, P. Advances in Star-Shaped $\pi$-Conjugated Systems: Properties and Applications. Macromol. Rapid Commun. 2014, 35 (11), 1006-1032

(7) Fischer, C. S.; Jenewein, C.; Mecking, S. Conjugated Star Polymers from Multidirectional Suzuki-Miyaura Polymerization for Live Cell Imaging. Macromolecules 2015, 48, 483-491

(8) Yuan, M.; Okamoto, K.; Bronstein, H. A.; Luscombe, C. K. Constructing Regioregular 
Star Poly(3-Hexylthiophene) via Externally Initiated Kumada Catalyst-Transfer Polycondensation. ACS Macro Lett. 2012, 1 (3), 392-395

(9) Tanaka, S.; Tanaka, D.; Tatsuta, G.; Murakami, K.; Tamba, S.; Sugie, A.; Mori, A. Concise Synthesis of Well-Defined Linear and Branched Oligothiophenes with NickelCatalyzed Regiocontrolled Cross-Coupling of 3-Substituted Thiophenes by Catalytically Generated Magnesium Amide. Chem. - A Eur. J. 2013, 19 (5), 1658-1665

(10) Xia, C.; Fan, X.; Locklin, J.; Advincula, R. C. A First Synthesis of Thiophene Dendrimers. Org. Lett. 2002, 4 (12), 2067-2070

(11) Bilge, A.; Zen, A.; Forster, M.; Li, H.; Galbrecht, F.; Nehls, B. S.; Farrell, T.; Neher, D.; Scherf, U. Swivel-Cruciform Oligothiophene Dimers. J. Mater. Chem. 2006, 16 (31), 3177-3182

(12) Karpe, S.; Cravino, A.; Frère, P.; Allain, M.; Mabon, G.; Roncali, J. 3D $\pi$-Conjugated Oligothiophenes Based on Sterically Twisted Bithiophene Nodes. Adv. Funct. Mater. 2007, 17 (7), 1163-1171

(13) Ma, C.-Q.; Mena-Osteritz, E.; Debaerdemaeker, T.; Wienk, M. M.; Janssen, R. A. J.; Bäuerle, P. Functionalized 3D Oligothiophene Dendrons and Dendrimers - Novel Macromolecules for Organic Electronics. Angew. Chemie Int. Ed. 2007, 46 (10), 16791683

(14) Wang, R.; Wang, W.-Z.; Yang, G.-Z.; Liu, T.; Yu, J.; Jiang, Y. Synthesis and Characterization of Highly Stable Blue-Light-Emitting Hyperbranched Conjugated Polymers. J. Polym. Sci. Part A Polym. Chem. 2008, 46 (3), 790-802

(15) Taranekar, P.; Qiao, Q.; Jiang, H.; Ghiviriga, I.; Schanze, K. S.; Reynolds, J. R. Hyperbranched Conjugated Polyelectrolyte Bilayers for Solar-Cell Applications. J. Am. Chem. Soc. 2007, 129 (29), 8958-8959

(16) Vanjinathan, M.; Lin, H.-C.; Nasar, A. S. Design, Synthesis, Photophysical, and Electrochemical Properties of DCM-Based Conjugated Polymers for Light-Emitting Devices. J. Polym. Sci. Part A Polym. Chem. 2012, 50 (18), 3806-3818

(17) Li, Z.; Ye, S.; Liu, Y.; Yu, G.; Wu, W.; Qin, J.; Li, Z. New Hyperbranched Conjugated Polymers Containing Hexaphenylbenzene and Oxadiazole Units: Convenient Synthesis and Efficient Deep Blue Emitters for PLEDs Application. J. Phys. Chem. B 2010, 114 (28), 9101-9108

(18) Kim, T.; Park, D.-K.; Ahn, T. Synthesis and Photophysical Properties of a Blue LightEmitting Hyperbranched Poly(fluorene). Mol. Cryst. Liq. Cryst. 2012, 567 (1), 125-131

(19) Han, Y.; Sun, M.; Fei, Z.; Bo, Z. Hyperbranched Polymer-Cored Star Polyfluorenes as Blue Light-Emitting Materials. Chinese Sci. Bull. 2008, 53 (18), 2770-2776

(20) Chen, J.; Peng, H.; Law, C. C. W.; Dong, Y.; Lam, J. W. Y.; Williams, I. D.; Tang, B. Z. Hyperbranched Poly(phenylenesilolene)s: Synthesis, Thermal Stability, Electronic Conjugation, Optical Power Limiting, and Cooling-Enhanced Light Emission. Macromolecules 2003, 36 (12), 4319-4327

(21) Wu, X.; Li, H.; Xu, B.; Tong, H.; Wang, L. Solution-Dispersed Porous Hyperbranched Conjugated Polymer Nanoparticles for Fluorescent Sensing of TNT with Enhanced Sensitivity. Polym. Chem. 2014, 5 (15), 4521-4525

(22) Richter, T. V.; Link, S.; Hanselmann, R.; Ludwigs, S. Design of Soluble Hyperbranched Polythiophenes with Tailor-Made Optoelectronic Properties. Macromol. Rapid Commun. 2009, 30 (15), 1323-1327 
(23) Roncali, J.; Leriche, P.; Cravino, A. From One- to Three-Dimensional Organic Semiconductors: In Search of the Organic Silicon? Adv. Mater. 2007, 19 (16), 20452060

(24) Steverlynck, J.; Leysen, P.; Koeckelberghs, G. Influence of Branching on the Chiral SelfAssembly of Poly(phenylene Ethynylene). J. Polym. Sci. Part A Polym. Chem. 2015, 53 (1), 79-84

(25) Ficker, J.; Ullmann, A.; Fix, W.; Rost, H.; Clemens, W. Stability of Polythiophene-Based Transistors and Circuits. J. Appl. Phys. 2003, 94 (4), 2638-2641

(26) Sun, Y.; Lu, X.; Lin, S.; Kettle, J.; Yeates, S. G.; Song, A. Polythiophene-Based FieldEffect Transistors with Enhanced Air Stability. Org. Electron. 2010, 11 (2), 351-355

(27) $\mathrm{Xu}, \mathrm{M} .-\mathrm{H} . ; \mathrm{Pu}$, L. Novel Unsymmetrically Hyperbranched Polythiophenes with Conjugation Gradient. Tetrahedron Lett. 2002, 43 (36), 6347-6350

(28) Scheuble, M.; Richter, T. V.; Goll, M.; Link, S.; López Navarrete, J. T.; Ruff, A.; Ruiz Delgado, M. C.; Ludwigs, S. Branched Polythiophenes by Ni-Catalyzed Kumada Coupling. Polym. Chem. 2014, 5 (23), 6824-6833

(29) Murakami, K.; Tanaka, S.; Mori, A. Linear-Selective Cross-Coupling Polymerization of Branched Oligothiophene by Deprotonative Metalation and Cross-Coupling. Polym. Chem. 2015, 6 (36), 6573-6578

(30) Scheuble, M.; Goll, M.; Ludwigs, S. Branched Terthiophenes in Organic Electronics: From Small Molecules to Polymers. Macromol. Rapid Commun. 2015, 36 (2), 115-137

(31) Takagi, K.; Torii, C.; Yamashita, Y. Polymerization of Branched Thiophene Monomers and Optoelectronic Properties of Materials. J. Polym. Sci. Part A Polym. Chem. 2009, 47 (12), 3034-3044

(32) Okamoto, K.; Housekeeper, J. B.; Michael, F. E.; Luscombe, C. K. Thiophene Based Hyperbranched Polymers with Tunable Branching Using Direct Arylation Methods. Polym. Chem. 2013, 4 (12), 3499-3506

(33) Yu, C.-Y.; Ko, B.-T.; Ting, C.; Chen, C.-P. Two-Dimensional Regioregular Polythiophenes with Conjugated Side Chains for Use in Organic Solar Cells. Sol. Energy Mater. Sol. Cells 2009, 93 (5), 613-620

(34) Park, J. W.; Lee, D. H.; Chung, D. S.; Kang, D.-M.; Kim, Y.-H.; Park, C. E.; Kwon, S.K. Conformationally Twisted Semiconducting Polythiophene Derivatives with Alkylthiophene Side Chain: High Solubility and Air Stability. Macromolecules 2010, 43 (5), 2118-2123

(35) Richter, T. V.; Braun, C. H.; Link, S.; Scheuble, M.; Crossland, E. J. W.; Stelzl, F.; Würfel, U.; Ludwigs, S. Regioregular Polythiophenes with Alkylthiophene Side Chains. Macromolecules 2012, 45 (14), 5782-5788

(36) Scheuble, M.; Gross, Y. M.; Trefz, D.; Brinkmann, M.; López Navarrete, J. T.; Ruiz Delgado, M. C.; Ludwigs, S. Polythiophenes with Thiophene Side Chain Extensions: Convergent Syntheses and Investigation of Mesoscopic Order. Macromolecules 2015, 48 (19), 7049-7059

(37) Lu, C.; Wu, H. C.; Chiu, Y. C.; Lee, W. Y.; Chen, W. C. Biaxially Extended Quaterthiophene- and Octithiophene-Vinylene Conjugated Polymers for High Performance Field Effect Transistors and Photovoltaic Cells. Macromolecules 2012, 45 (7), 3047-3056

(38) Smeets, A.; Van den Bergh, K.; De Winter, J.; Gerbaux, P.; Verbiest, T.; Koeckelberghs, 
G. Incorporation of Different End Groups in Conjugated Polymers Using Functional Nickel Initiators. Macromolecules 2009, 42 (20), 7638-7641

(39) Wu, S.; Huang, L.; Tian, H.; Geng, Y.; Wang, F. LiCl-Promoted Chain Growth Kumada Catalyst-Transfer Polycondensation of the "Reversed" Thiophene Monomer. Macromolecules 2011, 44 (19), 7558-7567

(40) Verswyvel, M.; Verstappen, P.; De Cremer, L.; Verbiest, T.; Koeckelberghs, G. Development of a Universal Chain-Growth Polymerization Protocol of Conjugated Polymers: Toward a Variety of All-Conjugated Block-Copolymers. J. Polym. Sci. Part A Polym. Chem. 2011, 49 (24), 5339-5349

(41) Kiriy, A.; Senkovskyy, V.; Sommer, M. Kumada Catalyst-Transfer Polycondensation: Mechanism, Opportunities, and Challenges. Macromol. Rapid Commun. 2011, 32, 1503-1517

(42) Sheina, E. E.; Liu, J.; Iovu, M. C.; Laird, D. W.; McCullough, R. D. Chain Growth Mechanism for the Regioregular Nickel Inititated Cross-Coupling Polymerisations. Macromolecules 2004, 37, 3526-3528.

(43) Yokoyama, A.; Miyakoshi, R.; Yokozawa, T. Chain-Growth Polymerization for Poly(3Hexylthiophene) with a Defined Molecular Weight and a Low Polydispersity. Macromolecules 2004, 37 (4), 1169-1171

(44) Miyakoshi, R.; Yokoyama, A.; Yokozawa, T. Catalyst-Transfer Polycondensation. Mechanism of Ni-Catalyzed Chain-Growth Polymerization Leading to Well-Defined Poly(3-Hexylthiophene). J. Am. Chem. Soc. 2005, 127 (49), 17542-17547

(45) Senkovskyy, V.; Khanduyeva, N.; Komber, H.; Oertel, U.; Stamm, M.; Kuckling, D.; Kiriy, A. Conductive Polymer Brushes of Regioregular Head-to-Tail poly(3Alkylthiophenes) via Catalyst-Transfer Surface-Initiated Polycondensation. J. Am. Chem. Soc. 2007, 129 (20), 6626-6632

(46) Doubina, N.; Ho, A.; Jen, A. K.-Y.; Luscombe, C. K. Effect of Initiators on the Kumada Catalyst-Transfer Polycondensation Reaction. Macromolecules 2009, 42 (20), 76707677

(47) De Winter, J.; Deshayes, G.; Boon, F.; Coulembier, O.; Dubois, P.; Gerbaux, P. MALDIToF Analysis of Polythiophene: Use of Trans-2-[3-(4-T-Butyl-Phenyl)-2-Methyl- 2Propenylidene]malononitrile-DCTB-as Matrix. J. Mass Spectrom. 2011, 46 (3), 237-246

(48) Liu, J.; Loewe, R. S.; McCullough, R. D. Employing MALDI-MS on Poly(alkylthiophenes): Analysis of Molecular Weights, Molecular Weight Distributions, End-Group Structures, and End-Group Modifications. Macromolecules 1999, 32 (18), $5777-5785$

(49) Doubina, N.; Stoddard, M.; Bronstein, H. A.; Jen, A. K.-Y.; Luscombe, C. K. The Effects of Binding Ligand Variation on the Nickel Catalyzed Externally Initiated Polymerization of 2-Bromo-3-Hexyl-5-Iodothiophene. Macromol. Chem. Phys. 2009, 210 (22), 19661972

(50) Willot, P.; Steverlynck, J.; Moerman, D.; Leclère, P.; Lazzaroni, R.; Koeckelberghs, G. Poly(3-Alkylthiophene) with Tuneable Regioregularity: Synthesis and Self-Assembling Properties. Polym. Chem. 2013, 4 (9), 2662-2671

(51) Verswyvel, M.; Monnaie, F.; Koeckelberghs, G. AB Block Copoly(3-Alkylthiophenes): Synthesis and Chiroptical Behavior. Macromolecules 2011, 44 (24), 9489-9498

(52) Watanabe, K.; Koyama, Y.; Suzuki, N.; Fujiki, M.; Nakano, T. Gigantic Chiroptical 
Enhancements in Polyfluorene Copolymers Bearing Bulky Neomenthyl Groups: Importance of Alternating Sequences of Chiral and Achiral Fluorene Units. Polym. Chem. 2014, 5 (3), 712-717

(53) Liu, J.; Zhang, J.; Zhang, S.; Suzuki, N.; Fujiki, M.; Wang, L.; Li, L.; Zhang, W.; Zhou, N.; Zhu, X. Chiroptical Generation and Amplification of Hyperbranched $\pi$-Conjugated Polymers in Aggregation States Driven by Limonene Chirality. Polym. Chem. 2014, 5 (3), 784-791 


\section{For TOC use only}

Joost Steverlynck, Fred Monnaie, Roberto Lazzaroni, Phillippe Leclère, Guy Koeckelberghs

Strategies towards controlling the topology of nonlinear poly(thiophenes)

Two series of branched poly(thiophenes) with different degrees of nonlinearity were prepared. In one approach the catalyst was changed for the polymerization of a branched $\mathrm{AB}_{2}$ monomer. In the other approach a copolymerization was performed with a linear monomer. Using different techniques the influence of the degree of nonlinearity on the morphology and self-assembly is studied.

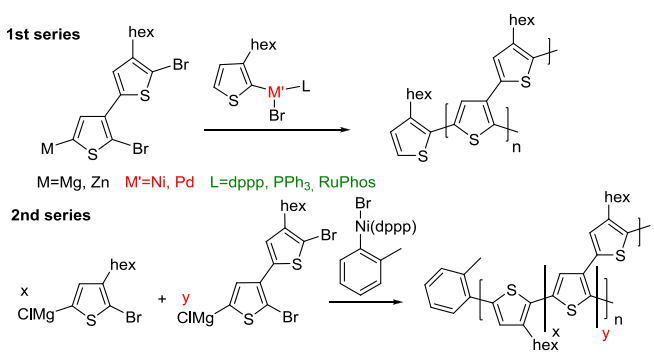

